\title{
Hand Made. Pour une anthropologie du geste musical
}

Jean During

\section{OpenEdition}

Journals

Édition électronique

URL : http://journals.openedition.org/ethnomusicologie/1834

ISSN : 2235-7688

Éditeur

ADEM - Ateliers d'ethnomusicologie

Édition imprimée

Date de publication : 1 décembre 2001

Pagination : $39-60$

ISBN : 2-8257-07-61-9

ISSN : 1662-372X

\section{Référence électronique}

Jean During, « Hand Made. Pour une anthropologie du geste musical », Cahiers d'ethnomusicologie [En ligne], 14 | 2001, mis en ligne le 04 avril 2012, consulté le 01 mai 2019. URL : http:// journals.openedition.org/ethnomusicologie/1834

Ce document a été généré automatiquement le 1 mai 2019.

Tous droits réservés 


\title{
Hand Made. Pour une anthropologie du geste musical
}

\author{
Jean During
}

\section{Prologue : histoire d'A. Le geste absolu}

1 Un jour dans une petite école persane, les élèves recevaient leur première leçon d'écriture. En retenant leur souffle et en serrant les dents, les doigts crispés sur leurs calames, ils tracèrent du mieux qu'ils purent la première lettre de l'alphabet (alif bâ) : l'A, alif. C'est un trait vertical tiré de bas en haut, simple comme le chiffre 1 (qu'il sert aussi à noter) et, pour cette raison aussi, la première lettre du nom divin. Les enfants s'appliquent à reproduire ce symbole de l'unité en alignant des rangées de traits parallèles sur les pages de leurs cahiers de classe. L'un deux y prend un soin et un plaisir extrême, et n'arrête pas de remplir des pages. Le maître passe maintenant à la lettre $\mathrm{B}$, mais sa forme de barque agrémentée d'un point n'intéresse pas le garçon, fasciné par la perfection du A. Il s'obstine à remplir ses cahiers de alif, et ne veut rien apprendre d'autre. Il finit par être chassé de l'école et demeure ignorant et analphabète, mais sans jamais cesser de tracer des A partout et à tout moment. Un jour, au hasard de son errance, il passa devant l'école et son maître le reconnut. Pour l'édification des élèves, celui-ci voulut leur présenter ce cas d'échec scolaire patent : «Eh toi, montre-leur donc tout ce que tu as appris à l'école », lui lança-t-il. Alors l'homme prit un bout de craie et l'appliqua fermement, de son bras tendu, sur le mur de la classe. Or voici qu'à mesure que la craie glissait sur la pierre en traçant une parfaite droite blanche, sous l'implacable détermination de son geste, le mur se fendit en deux.

2 Cette parabole, explique un éminent dépositaire de la tradition du Lorestan, s'applique à merveille à nos anciennes mélodies populaires: avec une lettre, un mot une simple ritournelle, elles vous transpercent le cœur, tandis que les autres musiques, avec toute leur science et leur art, ne parviennent pas à vous toucher autant. 


\section{Introduction}

3 Certes, mais une autre interprétation est permise : c'est bien du geste qu'il est question dans cette anecdote, du geste qui, répété au fil des années, devient parfait et finit par faire craquer, par faire tomber les murs. Etre touché, y mettre sa touche, un toucher inimitable, autant d'expressions qui renvoient l'une à l'autre, à partir du geste. Dans les représentations que certains se font de l'art musical, les sons relèveraient de l'intériorité, et le geste de l'extériorité, de la surface. Bien que nécessaires, les mouvements du corps sont au service de l'oreille et de l'idée et n'ont pas à être pris en considération. C'est pourtant par le geste que le corps s'implique dans la musique, si bien qu'elle prend corps et devient performance. Et tout naturellement c'est aussi par le geste que la musique exerce son impact et appelle des réponses physiques, ne serait-ce que les applaudissements permis à la fin.

4 Il existe donc deux positions bien tranchées, l'une idéaliste, opposant l'âme et le corps, l'autre, qui est plus couramment celle des musiciens, pragmatique, intégrant le corps. On les repèrera dans les pages qui suivent, à travers des exemples provenant des cultures musicales de l'Asie intérieure, notre champ d'étude. Au sein de l'aire parcourue ici, il est possible de dégager des oppositions pertinentes correspondant à différentes traditions et groupes ethniques, en particulier entre nomades et sédentaires. Cet aspect de notre étude retiendra peut-être l'attention des anthropologues dont l'un des plus fameux a lancé le débat en affirmant que l'homme est intelligent parce qu'il a une main.

5 Par ailleurs, l'analyse de près et sous différents angles de la posture, du toucher, du geste et de sa grammaire, aura des chances d'intéresser les musiciens de tous horizons. A travers les exemples présentés se dessine une typologie qui demande à être affinée et étudiée en tenant compte de ce que disent les maîtres de l'art. On distingue ainsi : le geste qui découpe le temps, ou au contraire, le fait fluer ou fluctuer, celui qui engendre des timbres, des figures, des motifs; le geste expressif, figurant le sentiment ou le mouvement mélodique; le geste héroïque du barde épique ou acrobatique du virtuose ; ou encore le geste imageant, allusion à un thème dans une composition « à programme "; enfin, avec les Kazakhs et les Kirghiz, apparaît le geste libre, qui s'affranchit du diktat de l'exécution musicale, qui prend les commandes pour faire sa musique à lui et qui pour bien signifier son autonomie, esquisse une danse des mains entraînant éventuellement l'instrument avec elles: bref, le geste qui réduit la musique au silence, comme pour rappeler, de la tête ou de la main, laquelle est le maitre.

\section{La part du corps}

6 La première leçon de musique commence par l'apprentissage de la position. Point de bon geste, point de beau son sans la position et la tenue d'instrument adéquates. Même le chant a sa posture et ses mouvements thoraciques propres. Un contact intime avec l'instrument est explicitement préconisé dans certaines traditions. Les joueurs de dotâr ${ }^{1}$ $\mathrm{du}$ Khorassan tiennent souvent l'instrument à l'envers, afin disent-ils, que la caisse soit tout près de leur cœur ( ( pour bien jouer de la guitare, disait Andrès Ségovia, il faut la serrer contre son cœur »). La tenue du târ azerbaïdjanais demande également un contact très physique qui semble viser l'efficacité maximale. On en joue en le serrant contre la poitrine, la caisse reposant sur l'avant-bras droit. Cette position établit une relation 
d'intimité avec l'instrument accentuée par le fait que l'oreille est plus près de la caisse. Malgré sa difficulté, elle est considérée comme essentielle à l'expression propre à cette musique : elle amplifie le volume du târ (peut-être parce que la poitrine fait office de caisse de résonance), elle permet de secouer tout l'instrument, de coller l'oreille dessus, de tordre le manche, de balancer le buste, de pivoter tout en jouant, voire de marcher avec, comme le font les chanteurs ouzbeks ou tadjiks. Les Azéris attachent une grande importance à cette position et méprisent la façon décontractée dont les Iraniens posent leur târ sur la cuisse, comme une guitare. Dans leur fierté, ils ne voient pas que ces derniers y trouvent leur compte, et que c'est délibérément qu'ils ont délaissé l'ancienne position. La douceur de timbre et la finesse de jeu qui en résultent correspondent, au dire des connaisseurs, à un retour progressif du style persan, marqué durant une époque par le goût turc caucasien².

7 Mais la différence essentielle ne se situe pas là. Elle relève d'un sentiment de soi, d'une image du corps totalement différents, qui se manifestent clairement entre des cultures bien distinctes, malgré les contacts étroits qu'elles ont eus. Ces deux positions, avec les éléments cinétiques et les attitudes qui en découlent, correspondent à un clivage qui se décèle à d'autres niveaux de la gestuelle, de la technique et finalement de la musique, entre d'une part le monde türk ${ }^{3}$ et de l'autre le monde indo-iranien, et d'une façon plus pertinente encore, entre cultures nomades et sédentaires.

8 On relève en effet une tendance générale à l'immobilité parmi les musiciens de culture sédentaire, non seulement en Iran, mais en Asie centrale. Abol Hasan Sabâ, selon l'expression de son disciple Dariouche Safvate ${ }^{4}$, était impassible comme un Bouddha. L'image vaut aussi pour le maitre de Tachkent Turgun Alimatov lorsqu'il joue le tanbur ou le satô. Asgar Bahâri, le joueur de vièle (kamânche) était si placide qu'il laissait se consumer sa cigarette sans tirer une seule bouffée durant toute sa performance. C'était un bonheur de plus de contempler durant des longues minutes les volutes de fumée glisser sur son visage ridé et ses yeux fermés, tandis qu'en l'absence de tout mouvement, la cigarette se transformait en un tube de cendre qui semblait attendre la fin du morceau pour se détacher du mégot. Avec Sa'id Hormozi c'était comme si le corps entier, fusionnant avec le setâr, finissait par l'absorber, limitant le geste au minimum concevable, tandis que la musique jaillissait à un débit intense. D’ailleurs, dès qu'il commençait à jouer, il abaissait la tête et semblait s'endormir. Lorsqu'il avait fini, il rouvrait ses yeux embués comme s'il sortait d'un rêve et restait silencieux. Quelques allusions de sa part suggèrent qu'il entrait dans un profond état de contemplation.

9 La plupart des musiciens iraniens s'efforcent de garder une apparence impassible sur scène ou à la télévision. Durant leur apprentissage, il est fréquent qu'ils s'appliquent à réduire le geste au minimum, sans pour autant sacrifier à l'efficacité. S'ils finissent par opter pour une technique naturelle, sobre mais sans inhibition, la plupart réduisent au minimum la participation du corps, au point que, sur scène, ils échangent à peine un regard entre eux. Cette réserve, qui est aussi imposée au public, ne peut s'expliquer par les seules contraintes du puritanisme islamique, même si elle opère une distinction vitale entre musiques sérieuse et non sérieuse (et donc méprisée), ou sert à se démarquer des traditions voisines arabe et azéri. Du reste, les rares représentants de l'ancienne école de chant (Sodeyf, Farahâni) soulignent l'expression mélodique et poétique par des mouvements des mains amples et variés. Il y a toutes les raisons de penser que la mise entre parenthèses du corps reflète un ensemble de valeurs, telles que la sacralisation $d u$ 
répertoire, la retenue des sentiments et la maîtrise de soi, les connotations mystiques, le sens du rang hiérarchique à tenir, etc.

Il en va tout autrement chez les Türks d'Orient et même chez les voisins Azéris qui sont pourtant de culture urbaine et sédentaire ${ }^{5}$. Alim Qasimov, pour ne citer que le plus connu des chanteurs de mugham azéri, ne tient pas en place, il tient en main un tambour sur cadre dont il se sert dans les parties non mesurées comme d'un porte-voix, et lorsque ses mains sont libres, il a des gestes qui rappellent ceux de Nusrat Fateh Ali Khan.

Le fameux joueur de saz azéri Edalat Nasibov change constamment de position de jeu comme pour suivre les séquences ou les phrases de ses airs : un moment il tient le manche à la verticale, un autre à l'horizontale, une fois il colle son oreille contre la caisse, une autre contre la table. D'une façon générale, le barde azéri (ashiq) se tient debout et affectionne les tenues militaires caucasiennes (casaque, ceinture de cuir, bottes, toque d'astrakan). Kanysh Zaynov, de Shamakhy, marche au pas de l'oie au rythme des percussions, tout en chantant et jouant du saz. Tout d'un coup, à la fin d'un motif à six temps rapides marqué par les percussions, il lance son saz en l'air de façon à le faire tournoyer sur lui-même, sans cesser d'avancer. Deux mètres plus loin, exactement sur le temps fort de la mesure suivante, il rattrape son instrument au vol et embraye sans aucun hiatus.

12 Ce genre de geste n'est pas toujours réductible à une démonstration de virtuosité. Il arrive qu'il traduise une forte émotion, un hâl. Le dotâriste et barde khorassanais Hâjj Qorbân Soleymâni lance son dotâr en l'air lorsqu'il entre en extase. Ostâd Elâhi, le maître sans égal du tanbur kurde faisait aussi ce geste dans sa jeunesse, dans la solitude de ses méditations musicales. Ces exemples nous rappellent que d'une manière générale, le modèle sédentaire vs nomade ne fonctionne pas dans le domaine mystique. Le derviche conçoit la vie comme un passage, un transit au point que certains font du nomadisme une règle de vie. Inversement, le chamane voyage sur place, selon un axe vertical. Tous deux mettent leur corps à rude épreuve: danse, exercices respiratoires, zikr, mouvements harassants, toujours en musique, si l'on peut dire. A ces exceptions près, la participation du corps, la gestuelle ostentatoire (mais aussi fonctionnelle et esthétique) des joueurs de luth nomades, repose elle aussi sur des valeurs. Elles se développent sur un fond épique exaltant la bravoure, l'efficacité, la vitesse, l'habileté.

Rendre compte de ces cultures en ces termes est un truisme, mais il faut bien le reconnaître : en Asie intérieure, les sédentaires se posent calmement pour faire de la musique, tandis que les nomades affectionnent le mouvement. Comme pour rétablir l'équilibre, les sédentaires usent largement de percussions et dansent avec tout le corps. Quant aux nomades (à part les chamanes), ils n'utilisent pas de percussion, ne dansent pas ou, à la rigueur, dansent assis, avec les mains et les bras, tout en jouant du luth ou en chantant $^{6}$. On tentera de décrire leur art à la fin de cet article. Avant cela, on procèdera méthodiquement, en abordant la question du geste à travers des données simples dont il est plus aisé de rendre compte.

\section{Le geste qui découpe le temps}

La musique baloutche ne se conçoit pas sans un accompagnement rythmique très présent, joué sur le tanburag, un luth à long manche au son puissant et au spectre acoustique étendu. On en frappe les cordes de haut en bas avec l'index, le majeur et l'annulaire 
groupés, et de bas en haut avec le pouce, selon un schéma des plus courant. Les cordes, très tendues, accordées en quarte (mi-la la), ne sont pas jouées à vide mais touchées de la main gauche de façon à produire un ostinato mélodique de base, du genre sol\# la mi / sol\# la mi. Sur ce bourdon rythmique (en 3, 4, ou cinq temps), la vièle (sorud) déroule des mélodies de structure simple mais ornées de façon raffinée, dense et variée ${ }^{7}$. Les mélodies se rangent en deux catégories: profane (chansons) et de transe (guâti damâli). Dans le genre de transe, le rôle du tanburag est plus important, car l'auditeur (chamane ou patient) est extrêmement attentif à tous les détails de la performance : il recherche une inflexion mélodique (l'air qui déclenchera la transe), une sonorité (il s'approche tout près du sorud pour mieux entendre), un rythme (qui le fasse se mouvoir ou danser) ${ }^{8}$.

$\mathrm{Au}$ cours d'une séance animée par un brillant sorudiste qui chantait tout en jouant, le patient était déjà en transe depuis une demi-heure lorsque le joueur de tanburag voulut passer le relais à un autre accompagnateur. Celui-ci jouait bien, mais sans égaler le premier. Ce détail, insignifiant pour l'auditeur, affecta le patient en transe. Sans cesser de s'agiter, il lui arracha l'instrument des mains pour le remettre dans celles du premier accompagnateur. Ce dernier demanda grâce au patient, invoquant la fatigue et son épaule douloureuse. Rien n'y fit. Au cours de la soirée, à chaque fois que celui-ci essayait de se faire remplacer, le patient lui remettait le tanburag dans les mains. Pendant tout ce temps, les mélodies et les rythmes changèrent souvent, ce qui suggère que la transe n'était pas tant guidée par la vièle que par le tanburag.

Cet exemple pose la question du geste musical à un niveau élémentaire, mais essentiel. La différence entre le bon accompagnateur et le moins bon n'était pas dans la tenue du rythme, mais dans l'effet acoustique de la frappe des cordes, de laquelle semblait dépendre la transe. Le tanburag est un instrument des plus simple, encore que ses formules rythmiques soient subtilement irrégulières. Pour en tirer le meilleur parti, il faut frapper les cordes avec détermination et force, tout en ménageant son effort, car il n'est pas facile de jouer plusieurs heures d'affilée, avec juste quelques brèves pauses (une mauvaise frappe peut littéralement blesser les doigts jusqu'au sang).

Le jeu du tanburag a peut-être un rapport avec le métier qu'exercent les tribus d'où sont originaires les musiciens : la ferronnerie. Certains musiciens sont aussi forgerons et, quand ils jouent, on plaisante volontiers sur leur façon de gratter les cordes du luth comme s'ils donnaient du marteau sur une enclume. En l'occurrence, le rythme et le geste sont comparables. De plus, les meilleurs musiciens ne reposent pas l'avant-bras sur l'éclisse du luth, comme c'est l'usage sur ce genre d'instrument, si bien que c'est avec le poids du bras tout entier qu'ils frappent les cordes.

\section{Les secrets du toucher}

Cette anecdote renvoie autant au sens du rythme qu'au toucher qui est, lui aussi, un aspect important de nombreuses pratiques musicales.

Dans le Khorassan iranien et afghan, l'instrument roi est le dotâr', un luth proche du tanburag baloutche, mais au service d'un répertoire mélodique en solo ou accompagnant le chant. Un jour à Torbat-e Jâm, deux jeunes gens en jouaient à tour de rôle, tous deux fort bien, à ceci près que l'un avait un son nettement plus lourd et plus plein. Lorsqu'on lui demanda pourquoi l'autre avait un son différent, il répondit d'un air entendu : « c'est bien cela qui fait la différence entre lui et moi ». Vingt ans après, il était devenu sans 
conteste le meilleur dotâriste de la région. La renommée de certains guitaristes de flamenco tient autant à leur toucher qu'à leurs autres qualités. Pour l'obtenir, ils se fabriquent de faux ongles semblables à des griffes. Le barde turc Hâjj Qorbân Soleymâni explique qu'il faut venir l'enregistrer au printemps, lorsque ses mains sont bien calleuses à force de tenir la charrue et de manier la bêche. Ainsi la sonorité de son dotâr gagne en puissance et en rondeur.

On demanda à Svjatoslav Richter ce qui l'avait le plus frappé dans le jeu de Neuhaus, le pianiste légendaire, avec qui il travailla à Moscou alors qu'il était déjà lui-même un maître. Il ne mentionna rien d'autre que la sonorité: sous les mains de Neuhaus, l'instrument sonnait d'une façon inouïe. Par une pertinente symétrie, ce qu'il a vraiment appris auprès de lui, c'est l'importance du silence dans la musique.

Le secret n'est donc pas ici dans la musculature et la consistance des doigts, mais, jusqu'à un certain point sans doute, dans le geste. Or le bon geste est aussi difficile à expliquer qu'à reproduire. La Strasbourgeoise Marie Jaëlle fut la première à tenter de visualiser le toucher. Disciple choyée de Liszt, elle était très attentive au beau son, dont la perception était, selon elle, la base de toute démarche musicale. Sa doctrine se résumait à ces mots que Liszt lui rappelait toujours : «à bon entendeur, salut ». Afin de sensibiliser ses élèves elle leur faisait poser les doigts sur un tampon encreur, puis, après qu'ils aient joué un motif, elle examinait les traces laissées sur les touches. Ainsi apparaissait le rapport entre la netteté de la trace et la beauté du son. Comme l'élégance d'une démonstration mathématique, le bon geste se mesure à l'économie de l'effort et s'apprécie à la grâce du mouvement et à l'efficacité du résultat.

Une interprétation moins "scientifique » est donnée par les derviches de la confrérie qâderi du Kurdistan en des termes rejoignant la théorie des arts martiaux d'ExtrêmeOrient. Il s'agit, disent-ils, de mobiliser une force vitale (sans doute l'équivalent du qi chinois), située au niveau de l'estomac, de la faire passer par le bras pour la libérer au moment précis où les doigts touchent la peau du tambour sur cadre (daf). Cette énergie est véhiculée par la vibration sonore et touche l'auditeur auquel elle transmet son effet. Il est probable qu'elle réveille à son tour une énergie du même ordre, favorisant une modification de l'état psychique. L'essentiel de l'art du chanteur et du percussionniste tient à la circulation de cette énergie ${ }^{10}$. Même s'ils en parlent autrement, les musiciens expérimentés sont probablement conscients de ces phénomènes.

Le toucher de l'instrument, comme le timbre de la voix, fait surgir d'emblée la dimension esthésique de l'émotion musicale. Il révèle dès les premières notes le charisme du musicien, sa faculté de captiver l'auditeur, le degré d'intimité qu'il a établi avec son instrument (ou sa propre voix). Il laisse deviner la profondeur, la concentration et l'intensité avec laquelle l'artiste s'est impliqué durant des années. Les degrés en sont variés. Pour aller plus loin que l'histoire du alif, Mollâ Nasreddin dirait peut-être que la perfection du toucher, c'est de faire sonner l'instrument tout seul. On raconte parfois des anecdotes de ce genre: les sonailles du luth gnawa (gumbri) qui frémissent lorsque les esprits sont là, la cithare du maître Burundi qui semble danser en lévitation devant lui tandis qu'il en pince seulement les cordes, le chant qui s'élève de la tombe d'un saint...

D'Ostad Elâhi, le maître charismatique du tanbur kurde ${ }^{11}$, les connaisseurs disaient: "Donnez-lui un morceau de bois sec, il pourra encore en tirer les sons du tanbur». Son fils, qui a hérité les secrets de cet art spirituel et ésotérique, en témoigne ainsi :

«Mon premier tanbur avait une sonorité lamentable et personne n'avait envie d'en jouer. Un jour, j'en fis part à Ostad et en demandai un autre. 'Comment ça, il sonne 
mal ?' dit-il, 'montre-le moi'. Il le prit et se mit à jouer : quelle sonorité ! On eût dit un orchestre entier... Jamais plus cet instrument n'a pu produire un tel son.

«Un jour, je l'ai vu jouer de son setâr en plein air, accompagné par d'autres instruments, dont des percussions, et par le chœur d'une grande assemblée battant la mesure avec les mains. Or, malgré tout ce volume sonore, c'était comme si son instrument dominait l'ensemble. [...] Dans ma naïveté, je me dis alors que cet instrument-là était fabuleux et j'eus envie à mon tour de le faire sonner de la même façon. Mais quand je le touchai, il n'en sortit qu'un tout petit son... $»^{12}$.

Figure 1 : Aygul Ulkenbaeva au cours d'une performance de dombra kazakh

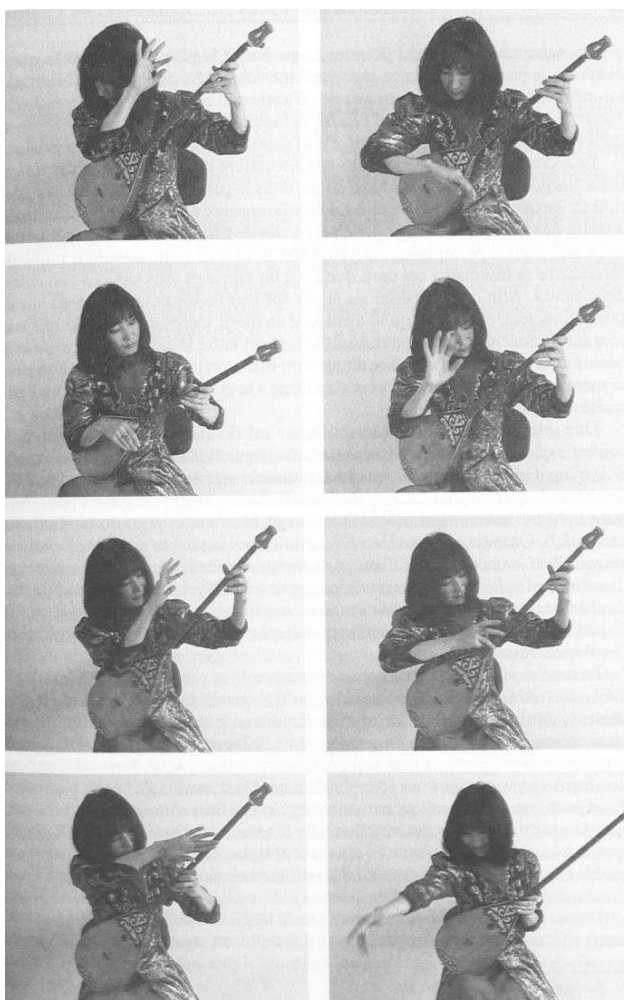

Photo : Jean During

\section{Le geste minimal}

On ne s'aventurera pas plus loin dans le domaine du sacré ou du surnaturel où tout semble possible tandis que rien n'est explicable. On s'en tiendra ici à ce qui peut se constater, en tentant de comprendre ce qui se passe.

Quel est le secret de Rasulbakhsh, musicien baloutche sans rival, pour que, quel que soit le sorud qu'on lui présente, il en tire les sons les plus suaves jamais produits par une vièle? Dans l'école russe de violon, on dit qu'un sérieux embonpoint, entraînant le déplacement du centre de gravité de toute la personne vers le bas, se traduit par un notable embellissement de la sonorité. Peut-être, mais notre virtuose baloutche est loin d'atteindre la corpulence de David Oïstrakh. Observons donc de près ses gestes et les mouvements de ses mains. Son secret n'est pas tant dans le soin qu'il met à l'accordage des cordes sympathiques (car tous les Baloutches sont très exigeants sur ce point), que dans sa façon de toucher les cordes et de faire glisser l'archet. Ce n'est plus la force qui est en jeu ici, mais une hypersensibilité tactile. Les crins ne sont pas tendus comme sur un 
archet de violoncelle, ce sont les doigts qui, par leur contact sur la mèche, assurent sa tension. Ce contact permet aussi de moduler finement les accents et les relâchements. Les mouvements sont courts et précis afin de mettre en valeur certaines notes. On ne tire ni ne pousse l'archet de façon linéaire et régulière, comme sur la plupart des instruments du genre : par instants, le crin flotte au-dessus des cordes sans plus les toucher; d'autres fois il s'abat sur elles par un geste de haut en bas. Faute de saisir ces nuances, le meilleur gambiste ou kamânchiste (joueur de kamânche, vièle courante en Asie Intérieure) ne tirera d'un sorud baloutche que des sons criards. En regardant une vidéo d'un de ses éminents collègues, Rasulbakhsh fait remarquer que le mouvement de son archet est légèrement plus ample que dans son école; il veut dire moins parfait que dans sa famille.

Le jeu de la main gauche se caractérise aussi par l'extrême sobriété des mouvements. Là aussi, on distingue tout de suite les amateurs de ceux qui appartiennent à ces lignées de musiciens qui se transmettent depuis des siècles les secrets de l'art. Chez ces derniers, les doigts touchent les cordes avec fermeté ou même les accrochent, alors qu'aucune force n'est requise pour faire sonner la note. En même temps, la pression est souvent relâchée (parfois avec un rapide glissando vers le haut) de façon à raccourcir quelque peu la résonance de la note, à la faire mourir une fraction de seconde. C'est dans ce petit intervalle de temps que les cordes sympathiques se font entendre en écho et que l'instrument ouvre un espace sonore d'une captivante richesse. Cette façon de lâcher la pression du doigt sur la corde se retrouve dans le rubâb afghan. Ici aussi, il s'agit de mettre en relief certaines notes, non seulement par des accents, mais par des moments d'extinction où les cordes sympathiques prennent le relais.

Ce principe était aussi appliqué par les maîtres du setâr persan comme Sabâ et Hormozi, dont les mouvements et déplacements de la main gauche étaient d'une sobriété fascinante. Dans certains traits ou sur certaines notes, aussitôt qu'il se pose sur la frette pour déterminer la hauteur de la note, le doigt se détend et la note meurt prématurément, tandis que l'on devine le son de la corde à vide un instant libérée. De nos jours, Dariouche Safvate est un des rares à posséder ce toucher particulier qui donne au setâr un caractère hésitant, humble, introverti, mais aussi subtil, riche et paradoxalement très précis. Ce maître, qui a toute sa vie médité sur le sens de sa musique, explique que les doigts doivent faire de petits mouvements, sentir les cordes et les caresser de façon à éprouver une jouissance dans chaque mouvement. Il donne volontiers l'exemple d'un oiseau qui picore le grain par de petits mouvements extrêmement précis et rapides. Par cette danse discrète des doigts, les deux mains, loin d'être une mécanique à domestiquer, prennent directement leur part de plaisir dans l'acte musical, ce qui est la condition préalable pour en procurer à l'auditeur.

\section{Excursus : la main du calligraphe}

Le toucher du setâr ou du târ, comme le glissement modulé de l'archet, présente des analogies avec la calligraphie, qui sont parfois relevées par les musiciens s'adonnant à cet art. Dans la calligraphie persane, comme chez les percussionistes, on définit un style, une patte, par des formules comme " main sèche ", " main grasse ", " main douce », etc. Mais plutôt que pour le toucher, c'est pour le geste et son rythme que l'analogie devient pertinente. En effet, le geste du calligraphe n'est pas continu : le trait module ses pleins et ses déliés (dus à la direction du trait, horizontal ou vertical, et non à la pression du calame), le mouvement ralentit par endroit, revient sur lui-même pour tracer un point, 
un accent, parfaire un raccord ou corriger le contour d'une ligne. Le geste est serré et d'une précision garantie par des normes : chaque lettre doit respecter les proportions canoniques mesurées en largeur du calame, sous forme de losanges. La liberté et la souplesse du trait sont régulées par des structures immuables, une sorte de trame que les maîtres de l'art ont sous la main ou dans l'œil à force de remplir des pages de alif, bâa, et autres djim. Sous les courbes de la calligraphie se devine la trame, tout comme sous les mélopées dites «libres" (âzâd) on perçoit un rythme, un flux, une respiration. Le chanteur respire au rythme du vers, comme le calligraphe retient et relâche son souffle au rythme du trait. Le calame lui-même n'est pas sans analogie avec la pointe de l'ongle ou le plectre frappant les cordes des luths setâr ou târ. Le travail d'enluminure aussi a son équivalent dans l'ornementation musicale : on tourne autour de la note, on suit le chant avec un léger décalage, comme en écho, à la façon des calligraphes qui brodent leur ligne d'écriture.

Ces analogies ${ }^{13}$ nourrissent certes le discours de tradition soucieux de dégager l'unité de la diversité des formes esthétiques, lesquelles se rattachent par ailleurs à une pensée holistique, à un système du monde. Elles prennent cependant un certain relief lorsqu'on examine leur équivalent dans d'autres cultures. Ainsi le geste musical, calligraphique ou plastique de la Perse, de l'Inde, de la Turquie ottomane, n'a rien à voir avec celui de la Chine ou des cultures nomades d'Asie centrale. Ici le plectre, le roseau taillé, le pinceau à un poil, serrés dans les doigts, là bas les cinq doigts, le pinceau mou et charnu tenu à main levée, le bras libre, le geste ample rapide, extraverti, qui se donne à voir jusqu'à la limite de l'ostentation. Enfin, chez les vrais nomades, pas d'écriture, mais une riche orature où le geste peut prendre une place essentielle ${ }^{14}$.

\section{Tour de main et flux rythmique}

Dans le tanbur sacré des Kurdes, et notamment dans l'école transcendante d'Ostâd Elâhi, la libération de la main droite, celle qui produit les sons, passe par sa mort symbolique. Le premier geste qu'on apprend est celui de faire tomber la main droite sur les cordes " comme un morceau de viande morte". L'image de la viande se comprend comme désossée, ce qui n'est pas évident à réaliser : relâcher toute tension, laisser la main trouver sa forme naturelle, laisser les doigts se placer, abdiquer toute volonté, puis, après la chute, remonter en laissant le pouce trouver tout seul sa place et sa fonction, qui consiste à frapper les cordes dans l'autre sens. Après les formules courantes telles qu'on les trouve dans le jeu du dotâr en général, si l'élève a la main assez souple, il commence à s'exercer à faire le shor: pouce, index, majeur, annulaire, auriculaire se repliant tour à tour à partir de la main grande ouverte jusque vers la paume, comme un éventail qui se ferme. Pas question de commencer avec un instrument, on s'exerce en posant la main sur la cuisse, en grattant un bord de table, puis en touchant les cordes, mais étouffées. Après cela, on peut s'essayer sur l'instrument: ouvrir et fermer l'éventail des doigts en effleurant les cordes à grande vitesse. Les débutants sont fascinés par cet apprentissage ; ils oublient tout le reste pour parvenir à faire un shor correct, comme si la philosophie ou la sagesse du tanbur tenait à ce geste. Ou peut-être tout simplement parce que le déploiement des doigts, s'il est bien fait, a la grâce d'un paon qui fait la roue. Si l'homme est intelligent parce qu'il a une main, c'est l'esprit lui-même qui s'exprime par ce geste.

Un détail retient notre attention: dans quasiment tous les luths de ce genre, la main droite monnaye les notes en 2 et en ses multiples. La forme de base, que l'on retrouve 
dans les instruments à plectre, est le coup descendant ou ascendant (droit-gauche, chap râst, ou le contraire ${ }^{15}$ ). Dans les doigtés plus complexes, trois ou quatre mouvements sont mis à contribution de façon à monnayer les notes en 3 ou 6 , ou en 4 et 8 . Ainsi toutes les mélodies se calent sur une trame binaire, parfois ternaire, finalement assez banale. Dans la technique élaborée et répandue par Ostâd Elâhi, le roulement met à contribution les cinq doigts de la main droite, si bien que les notes d'une certaine longueur sont monnayées en cinq, ce qui donne une fluidité incomparable à la mélodie, libérée de la banale symétrie du monnayage binaire ${ }^{16}$. Ce n'est pas une coïncidence si le terme désignant ce mouvement, shor, évoque le flux d'une rivière, d'une chute d'eau.

Le shor du tanbur illustre un principe fondamental de l'esthétique du monde iranien, qui s'étend de l'Azerbaïdjan au Xinjiiang : produire du continu à partir de discontinu. Il n'y a pas de percussion (zarb), de luth (setâr, dotâr, târ), de cymbalum (santur) qui n'ait son « roulement » ou «tremblement " propre (en persan, riz). La maîtrise du riz s'acquiert lentement et ne se maintient qu'avec une pratique régulière. Ici également, il ne s'agit pas de diviser les temps en 2 (croche), 3 (croche pointée) ou en 4 (noire), comme on l'enseigne dans les mauvaises classes de ' $u d$ arabe ou turc, ni non plus d'appliquer systématiquement des roulements continus de mandoline. Notre regretté maitre Ali Akbar Shahnâzi expliquait que le plectre du luth târ (mezrâb) devait être semblable à un archet pour cet instrument, oubliant de préciser qu'il s'agissait d'un archet de vièle orientale, comme celle de Rasulbakhsh (cf. supra). En effet, pour que le flux ne soit pas monotone, il est agrémenté de micro-ruptures, de tensions, de contournements, un peu comme le cours d'un ruisseau qui fait des tourbillons, qui roule sur les rochers, qui ralentit dans les méandres. Ainsi, les roulements sont irréguliers, variés et de vitesse modulable, selon des modèles qui correspondent à des écoles ou des styles personnels. Ici encore, l'analogie avec la calligraphie se justifie.

\section{L'accentuation}

Certains de ces mouvements ou tours de main peuvent être considérés comme des gestes « purs » en ce sens qu'ils se laissent difficilement appréhender de façon analytique, qu'ils débordent la représentation que l'on s'en fait. Si les interprètes ont bien dans l'esprit quelque modèle mélodico-rythmique précis, les détails de l'ornementation sont laissés à la main droite qui fait les notes comme à l'insu de l'œil et de la pensée. De fait, un luthiste ne regarde jamais sa main droite, mais plutôt sa gauche. Il est intéressant pour un musicien de remarquer qu'il joue certains roulements sans avoir une claire idée de ce que fait son index dans son aller et retour cyclique. Il ne se rend compte de la complexité de son geste que lorsqu'il doit le décomposer pour un élève ou le transcrire en notes. L'exercice n'est pas aisé, il met l'interprète dans la situation du mille-pattes qui trébuche en voulant expliquer comment il marche.

Dans certains cas, c'est la morphologie de la main du musicien qui lui permet de réaliser des formules rares et inimitables qui deviennent une composante de son style personnel. Dans la musique persane, la difficulté ne tient pas tant à la formule elle-même dans son alternance de coups droits et gauches, qu'aux différences de tension et d'accentuation au sein d'une formule. Le maître iranien Dariouche Safvate en donnait un exemple très simple : 


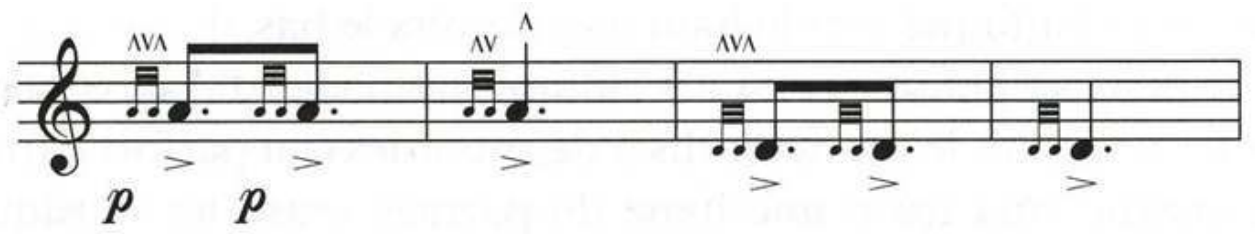

les deux premières notes doivent être jouées $p p$, et la troisième $f$. Il faisait remarquer que de fameux joueurs de setâr n'arrivent pas à réaliser ce geste si simple. Il sont réduits à «tricher » en ajoutant une appoggiature :

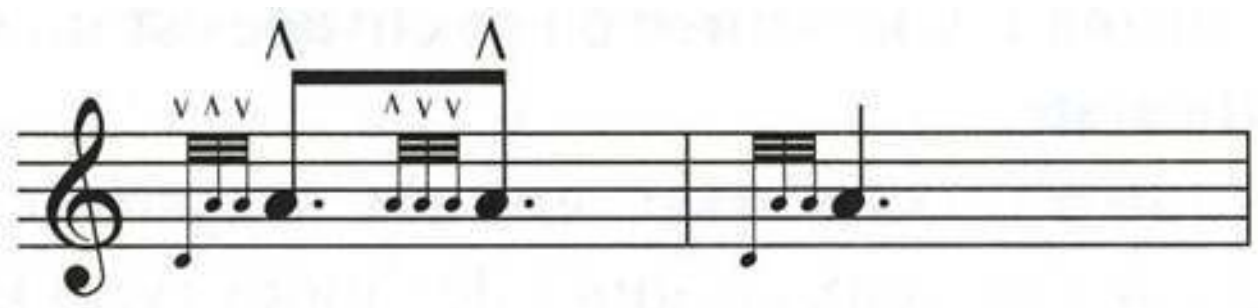
onglet est caractéristique des cultures sédentaires de l'Asie intérieure et du ProcheOrient. Elle reproduit, (ce n'est pas un hasard) le système des instruments de percussion dont l'usage est un trait propre à ces cultures. Cette technique, que l'on retrouve dans les 'ud, târ, rabâb, baglama et divers tanbur et sitar, engendre, on l'a vu, un système binaire qui prend en compte le léger contraste de timbre et d'intensité entre l'attaque vers le haut et celle vers le bas.

41 Ce type de geste s'oppose à celui des cultures nomades d'Asie centrale, tel qu'il apparait clairement dans le jeu des luths à deux cordes (ou parfois à trois). Le toucher de ces 
instruments trace une ligne de partage entre les musiques de type nomade et de type sédentaire, ou disons en simplifiant, entre Türks et Indo-Iraniens. (De ce point de vue, le tanbur kurde et le dotâr du Khorassan central se rattachent plutôt au premier groupe, ce qui se comprend par le contact étroit entre les Türks et les Kurdes ou Iraniens de la région, ainsi que par leur culture encore marquée par le nomadisme.) A la source de ce clivage est un simple geste musical d'une remarquable efficacité.

En effet, dans les luths türks d'Asie centrale, le système de frappe n'est pas binaire, mais ternaire, car aux deux attaques de l'index (vers le haut et le bas), s'ajoutent celle du pouce (vers le haut). Ceci a pour effet non seulement de doubler la vitesse sans aucun effort, puisque le rythme des allers et retours de la main (haut/bas) reste le même. Par ailleurs, l'utilisation d'un autre doigt (et plus encore, de tous les doigts) permet de varier les timbres. Alors que le plectre (ou l'index) fait son aller retour,

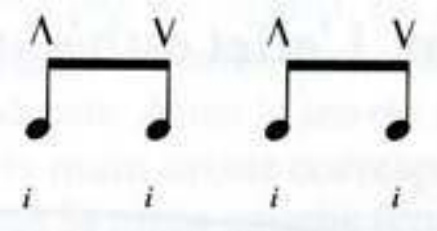

dans les luths à deux cordes, le pouce, par sa position naturelle effleure les cordes sans aucun effort de façon à monnayer la pulsation en :
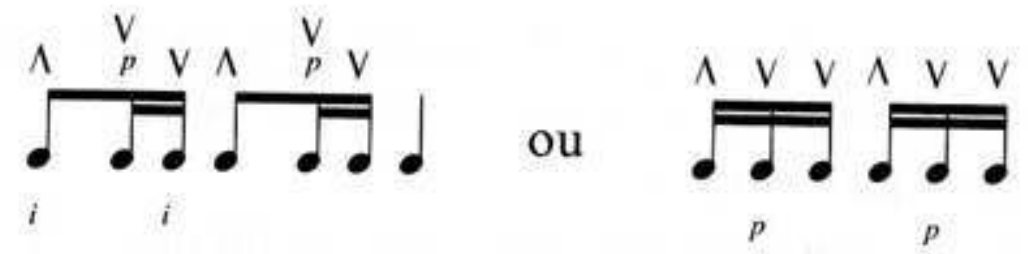

Lorsque le pouce à son tour se met a faire des aller-retour, la vitesse est doublée sans que le mouvement de la main s'accélère :

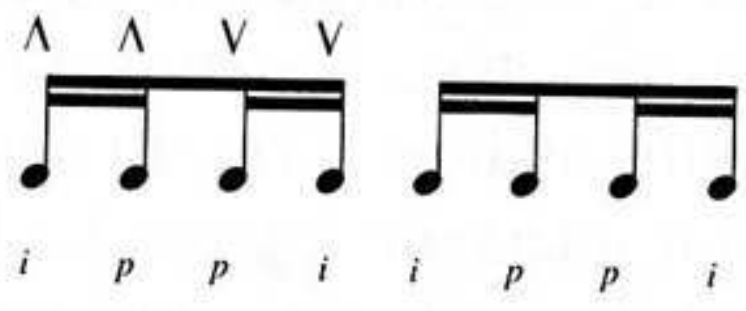

Avec trois mouvements au lieu de deux, une formule comme celle évoquée plus haut, difficile à réaliser au plectre ou à l'index, est des plus simple à jouer avec deux doigts.

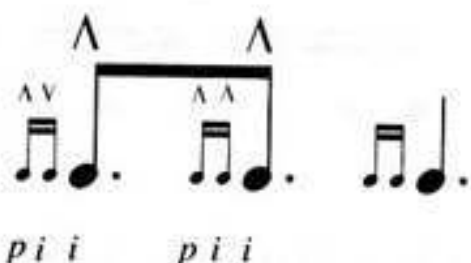

Il est probable qu'un certain nombre d'ornements, de tremolos, de figures gestuelles et rythmiques propres aux instruments à plectre comme le târ persan et âzeri, le tanbur tajik et ouzbek, le rabâb uygur proviennent des luths à cordes pincées par les doigts, du genre 
du dotâr, qui sont plus anciens ${ }^{17}$. Si ces ornements ne se retrouvent pas sur le 'ud arabe et turc, ou sur le tanbur turc, c'est peut-être parce que l'aire de la famille des dotâr se situe plus à l'est ${ }^{18}$.

47 A partir des trois mouvements élémentaires de l'index et du pouce, chaque tradition a développé sa technique en mettant à contribution les autres doigts de la main droite. Ainsi, il devient possible de faire des roulements extrêmement rapides avec trois doigts frappant dans deux directions, d'une façon qui rappelle curieusement le jeu de la guitare flamenca élaboré par les Gitans, un autre peuple d'origine nomade. De plus, dans le dombra kazakh et le komuz kirghiz, outre les frappes, on utilise la technique du pincé et du buté $^{19}$; un autre point entre le jeu de cette guitare et celui des dotâr est l'intégration de l'élément rythmique, au point que même dans les genres urbains et classiques (en Asie centrale), on considère comme redondante et indésirable l'adjonction d'une percussion sur le jeu des luths à long manche de la famille du dotâr ${ }^{20}$.

Tous les accents ou syncopes, monnayages ou nuances de timbre (sec, doux, résonnant, etc.) qui font l'intérêt d'un accompagnement rythmique (tambourin, ou autre) se trouvent intégrés dans le jeu du dotâr, avec plus de variété encore.

Il est aisé de faire l'inventaire des formules de base du jeu du rubâb afghan, du gumbri marocain, ou du qanbûs yéménite, avec leurs combinaisons de coups droits et gauches. Cela n'est pas très difficile dans le cas du dotâr khorassanais, où tous les coups de main se résument à faire intervenir le pouce $(\mathrm{v})$, l'index $(\wedge \mathrm{v})$ et les autres doigts groupés $(\wedge)$. Pour le dotâr ouzbek-tadjik, c'est une autre affaire: non seulement le pouce mais les autres doigts se meuvent dans les deux sens. Le roulement de base se fait non pas ${ }^{\wedge} \mathrm{v}^{\wedge} \mathrm{v}$ sur la base d'un aller-retour de l'index (dotâr khorassanais),

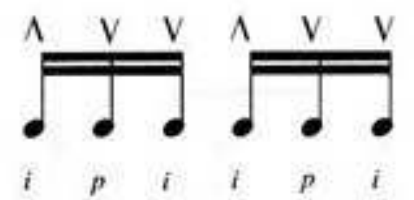

non pas $\mathrm{p} v / \mathrm{i} v / \mathrm{i}^{\wedge} /$, comme chez les Turcs et Kurdes du Khorassan, mais : $\mathrm{a}^{\wedge} / \mathrm{p}^{\wedge} / \mathrm{pv} /$ $\mathrm{i}$ /

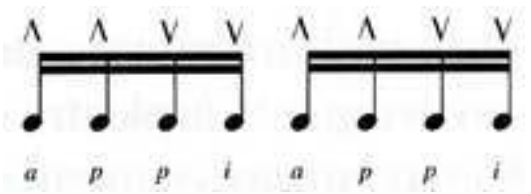

51 (une version plus simple se joue chez les Oügours: $\left.\right|^{\wedge} / \mathrm{p}^{\wedge} / \mathrm{pv} / \mathrm{iv}$ ). Ainsi, le simple roulement devient une affaire complexe mettant à contribution trois doigts dans deux directions. 


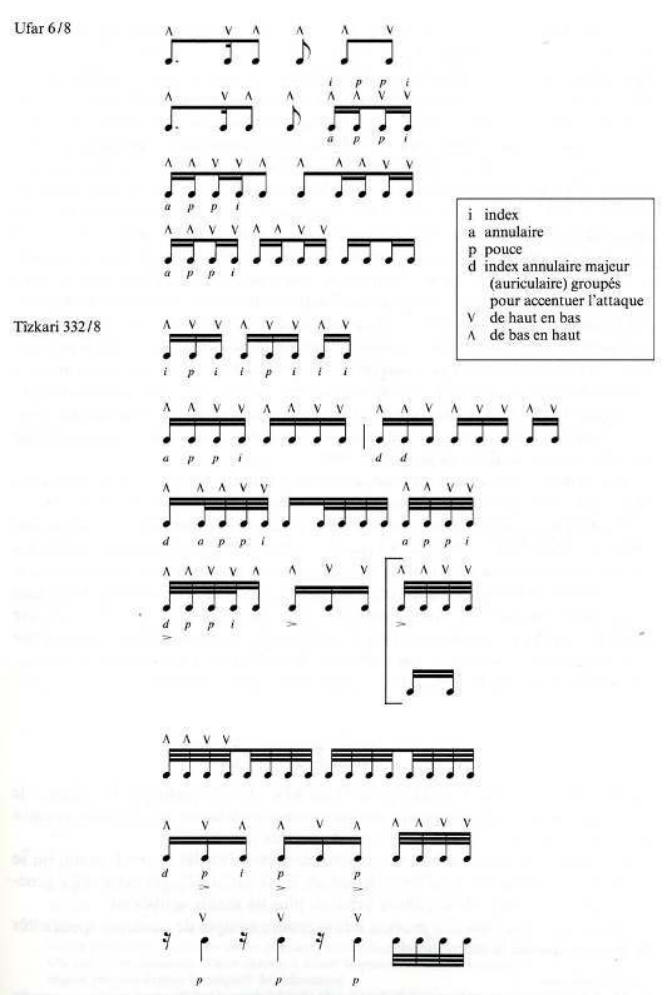

Sur ces bases, chaque école développe son propre style, utilisant tel ou tel doigt dans tel ou tel sens, en combinaison avec tel autre pour produire une variété de formules de remplissage dont il serait vain de prétendre faire un inventaire exhaustif. Bien entendu, certaines écoles se limitent à un petit nombre de formules : le dotâr afghan, khorassanais ou qâraqâlpâk, le dombra uzbek et tadjik, le dotâr ouïgour utilisent un nombre limité de formules rythmico-gestuelles (cf. During 1993 et 1997b). Il en va autrement du dotâr ouzbek dont la technique s'est développée au XIXe siècle afin d'intégrer le répertoire classique (maqâm). On trouve bien des méthodes de dotâr tadjik-ouzbek, mais elles ne prennent en compte que les frappes de base, les formules rythmiques standard telles que l'exemple ci-contre.

Dans le jeu artistique, la même phrase (mesurée) peut être réalisée de quatre ou même huit façons différentes mettant à contribution des patrons rythmiques ainsi que des doigtés variés. Il y a bien des recettes, des patrons courants, mais les maîtres ont, en plus, leurs doigtés à eux. Tenter d'en dresser l'inventaire ne les intéresse pas. Les connaisseurs se contentent de remarquer que tel interprète utilise par exemple l'annulaire au lieu de l'auriculaire dans un certain type de roulement, ou relèvent quelque autre particularité qui lui confère sa touche personnelle. Une fois dépassé le cadre académique, qui est celui de la représentation systématique et des méthodes de dotâr, libre à chacun de trouver d'autres doigtés, d'autres gestes. En ce sens, le jeu du dotâr (et plus encore du komuz kirghiz) excède toujours la représentation que l'on peut en faire. Leur technique se transmet par l'imitation, en regardant, en comprenant avec les mains et le corps, à l'instar de la danse. C'est cette richesse qui destine ces instruments au solo, en particulier le dombra kazakh et le komuz kirghiz qui ne se jouent qu'en solo, sauf lorsque l'interprète chante avec $^{21}$. Ces considérations valent pour d'autres instruments comme le zarb (tombak ) iranien dont le jeu et les techniques, requisitionnant les dix doigts, évoluent sans cesse. La moindre mélodie ouzbek jouée au dotâr prend une couleur particulière, variable en fonction de l'interprète, et ce d'autant plus que la main gauche y apporte son éclairage 
propre à base de quartes, quintes, octave, comme on l'expliquera plus tard. Si l'on peut écouter sans se lasser un concert ou un disque de dotâr ou dombra (cf. note supra), ce ne saurait être le cas pour d'autres instruments d'Asie centrale, comme la flûte ou la vièle en solo.

On en jugera avec cet exemple tiré d'un motif d'une pièce de dotâr du Khorezm, Qora dali, dont nous donnons ici quelques variantes possibles (voir pp. 58-59).

Nous n'irons donc guère plus loin dans l'analyse des techniques de jeu de ces différents luths. Brièvement, disons qu'ils s'étendent sur une échelle allant du plus simple: combinaisons de ${ }^{\wedge}$ et $\mathrm{v}$ dont la grammaire est aisée à cerner, jusqu'à des gestes extrêmement complexes qui n'ont plus aucune fonction acoustique, comme dans le jeu du dombra kazakh et plus encore du komuz kirghiz (cf. During 1997c). Entre ces deux extrêmes, on relèvera ici ou là des formules assez simples, typiques d'une école et absentes d'une autre : par exemple, - la répétition rapide de frappes vvvv... exclusivité des Kazakhs et Kirghiz ; - l'alternance peu commode :

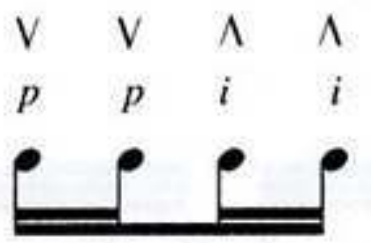

p̌p̌n̂i / p̌p̌n̂i chez les Kazakhs et les Oügours, - le shor propre aux Kurdes, - le chortme («nœud », qui renvoie au geste de la noueuse de tapis), ou « coup de ciseau » (qeychi) qui donne au dotâr Turkmène sa sonorité incisive.

Plutôt que de s'appliquer à répertorier ces innombrables tours de main, on se risquera, en comparant tous les styles de jeu du dotâr, à dégager cette règle générale : plus on s'éloigne de la culture urbaine, plus les mains se libèrent.

Avant d'en dire plus à ce propos, il faut rendre compte de quelques spécificités de la technique de la main gauche.

\section{Le double jeu nomade}

Le musicien nomade se distingue généralement de son homologue sédentaire en ce qu'il cumule différents talents et assume différents rôles. Ce dernier est soit instrumentiste, soit chanteur, soit percussionniste, et même si ses compétences sont multiples, il s'intègre volontiers à un ensemble instrumental partageant les rôles. Le barde de culture nomade, quant à lui, est à la fois chanteur, instrumentiste, conteur, poète, lettré, humoriste, historien, homme de pouvoir ${ }^{22}$, parfois chamane, et il se présente tout seul ${ }^{23}$.

Cette accumulation de compétences apparaît également dans le jeu de certains instruments essentiels comme les luths à deux ou trois cordes. Alors que dans les styles urbains et classiques on se contente de jouer une mélodie habilement ornée, généralement étoffée par d'autres instruments jouant dans un unisson relatif, ici, avec un seul instrument (auquel peut s'ajouter la voix), on superpose sous une forme très compacte différents niveaux : la mélodie, le rythme ${ }^{24}$ et l'harmonie. Le jeu des luths des Kazakhs et des Kirghiz, et dans une moindre mesure des Turkmènes et des Qâraqâlpâks est en quelque sorte hétérophonique, à base de quartes, quintes, tierces, etc. Il s'agit là d'une constante des musiques nomades : obtenir du multiple avec du simple. A l'appui de 
cette proposition, citons le chant diphonique qui donne deux sons avec une seule voix, la guimbarde ${ }^{25}$, les instruments à archet dont les cordes en crin produisent souvent des harmoniques ou des quintoiements, ainsi que les flûtes du genre ney (sybyzgy), avec lesquelles on chante un bourdon tout en jouant; enfin, le jeu de l'instrument le plus répandu, le luth à deux cordes, puisqu'on frappe toujours deux notes ensemble, avec les conséquences que nous verrons plus loin.

61 Cette constante dans le redoublement n'est pas sans rappeler quelques spécificités de la langue turque qui appartient comme on sait au groupe des langues agglutinantes. Ainsi la négation, l'interrogation, le pronom, le gérondif y sont groupés en une seule forme verbale. De plus, elles manifestent une propension à décrire une action en deux étapes, avec circonstant et prédicat, du genre "ayant (ou étant)... j'ai... $»^{26}$ L'action n'est pas séparée, mais elle est décrite en deux mots. De la même façon, sur un luth à deux cordes accordé en sol do, lorsqu'on veut dire ré, on joue sol-ré simultanément (une quinte) ; mais pour dire sol, on jouera probablement une octave, quant à sib, on le jouera fa-sib (quarte).

Exemple tiré d'un motif d'une pièce de dotâr du Khorezm, Qora dali, avec quelques variantes possibles

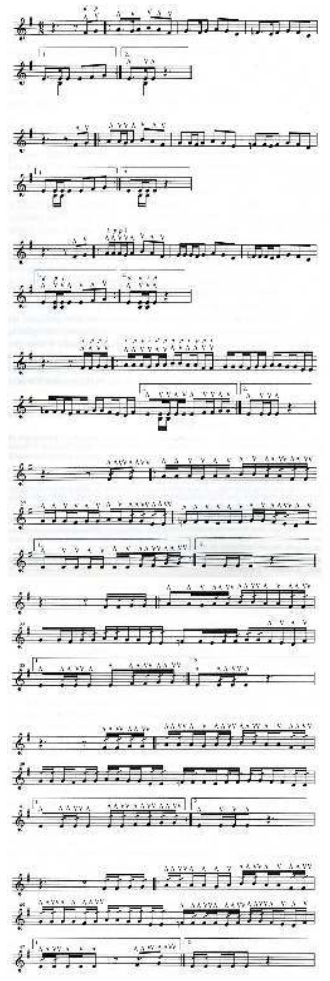

Ainsi, le geste de la main gauche est-il toujours double: tandis que les quatre doigts jouent ce que l'on peut appeler la mélodie principale sur la corde aiguë, le pouce se déplace plus ou moins parallèlement aux autres doigts, jouant sur la corde grave. Chez les Kazakhs, les doubles notes sont encore plus systématiquement utilisées. (De même dans leur langue, la combinaison gérondif + verbe est encore plus compacte et finit par donner un lexème indécomposable.) En dehors du pouce, d'autres doigts sont mis à contribution sur la corde grave pour entretenir la biphonie de la mélodie, comme sur une guitare qui n'aurait que deux cordes. L'origine de cette biphonie ne peut s'expliquer que par le geste, sans quoi on la retrouverait dans d'autres formes musicales. C'est le toucher de l'instrument, la gestuelle de la main gauche, qui en est la source. On le retrouve chez les 
peuples türks d'Asie centrale, et jusque dans les luths turcs d'Anatolie (sâz, baglama ou cura). En revanche il se perd dans les aires iranienne, tadjik, indienne et évidemment arabe. Il n'est pas lié à l'instrument, mais à sa technique et à la prise de la main sur le manche, car entre le style turkmène et khorassanais de l'est (ou de l'Afghanistan), le jeu du pouce sur la basse disparaît complètement.

Le glissement de style se fait sentir au sein d'une même musique et d'un même répertoire, entre le jeu du dotâr classique ouzbek et son homologue tadjik. Dans ce dernier, on use nettement moins du pouce pour doubler les notes de la mélodie et l'on distingue d'une part la mélodie, et de l'autre, des notes d'agrément dans le grave, alors que dans le jeu ouzbek, on enseigne les deux sons simultanément. La basse ne s'ajoute pas à la mélodie (qui pourtant existe séparément puisqu'on la chante), elle y est intégrée par le geste. Cette tendance nous apparut clairement lorsqu'il fallut enseigner une mélodie persane à une professionnelle du dotâr ouzbek: rien ne pouvait bloquer la machine gestuelle qui ajoutait à la mélodie les notes graves variables, formant des quartes ou des quintes parallèles. L'adjonction de ces notes confère curieusement une teinte centre-asiatique aux airs persans et les rend totalement irrecevables pour les Iraniens. Inversement, les jouer avec un bourdon constant (ou changeant ici ou là en fonction de la dominante), semble ennuyer les oreilles ouzbek. Ce clivage entre goût nomade et sédentaire d'Asie apparait encore mieux entre l'Asie centrale et l'Inde, où le bourdon est rigoureusement invariable ${ }^{27}$. Bien entendu, les Ouzbeks sont sédentaires comme les Tadjiks, mais depuis quelques siècles seulement, et par ailleurs, le dotâr représente la composante nomade de leur instrumentarium.

Si l'Inde préserve la forme radicale de cet art que nous appelons "sédentaire", son extrême opposé est l'art des Kazakhs et Kirghiz, que nous qualifions de «nomade ». Ce terme de doit pas être entendu dans son sens proprement ethnographique, mais comme un concept à résonance philosophique et esthétique. Le style nomade peut survivre longtemps à la sédentarisation tout en renvoyant à une appréhension du temps et de l'espace qui est bien celle du mode de vie des ancêtres ${ }^{28}$. Il apparait dans toute sa force dans le jeu de deux luths à long manche : le dombra kazakh (deux cordes) et le komuz kirghiz (plus court, à trois cordes et sans frettes).

Brièvement dit, pour nous en tenir à la composante gestuelle de cette esthétique, dans bien des cas, c'est comme si les pièces instrumentales (kui, kuu) procédaient par succession de positions ou de mouvements des mains, de "chiromorphèmes " pourraiton dire. Pour parler en « Deleuzien », on dira que les déplacements de la main dans les kui se font «de proche en proche $»^{29}$, empiriquement, de façon à remplir l'espace. Toute autre est la démarche, ou en l'occurrence, le mouvement de mains de l'esthétique sédentaire. Il s'agit là "d'occuper des positions » sur un axe orthonormé (puisque toutes les notes sont référées à une note fondamentale ou tonique) et, pour la main droite, de frapper les notes selon un "striage " rythmique homogène (la mesure, le cycle, le mètre poétique). Se placer, atteindre des positions et y rester (le terme maqâm lui même signifiant "position» ou «station»), travailler les notes en profondeur (par l'ornementation), les assiéger pour reprendre les images deleuziennes de «machine de guerre des sédentaires » est tout le contraire du geste nomade. Ici, le geste est rapide, le tempo est trépidant mais modulé (référence au galop du cheval, avec ses accélérations et ralentissements), les pièces se déroulent à la vitesse d'un raid, elles accumulent des effets, des figures, en une logique imprévisible (pas de cyclicité rythmique, pas de mètre 
poétique sous-jacent), et sont trop courtes (deux à cinq minutes) pour laisser le temps de dévoiler leur stratégie.

On ne reviendra pas sur les concepts deleuzien (et guattarien) de « lisse » et de "strié » qui rendent bien compte de ces deux esthétiques ${ }^{30}$; on esquissera seulement deux autres notions qui en dérivent : celles d'habileté et d'hétérogénéité.

\section{Habileté et virtuosité, hétérogène et homogène}

Rappelons d'abord que dans son sens originel, ars ne renvoie pas aux formes ou aux représentations, mais, bien plus concrètement, à l'habileté. Beaucoup de musiques offrent de beaux exemples où l'habileté est bien plus qu'une aptitude physique permettant de réaliser une idée: le geste dépasse la forme sonore qui le met en lumière comme un ornement, ou encore, la forme sonore elle-même apparait comme un geste, comme une tournure, un tour de passe-passe qui possède sa propre élégance ${ }^{31}$. Par geste, on n'entend pas seulement ici le mouvement de la main, mais aussi bien les doigtés, les trucs, toutes les astuces (les raccourcis de la pensée), dans la configuration d'un instrument qui permettent de briser l'uniformité ou la continuité, d'ouvrir des perspectives, de créer des articulations, de connecter des notes de manière originale, etc.

C'est dans la technique instrumentale que cette qualité apparaît plus clairement ; mais cette technique est elle-même conditionnée par l'habileté et l'astuce mises en jeu dans la conception de l'instrument, notamment la place des notes, la disposition des cordes, l'accordage. L'habileté, c'est par exemple l'accord croisé du luth maghrébin kwitra par rapport au 'ud classique, celui du komuz kirghiz (une douzaine d'accordages différents pour trois cordes). C'est encore la répartition des notes du balafon (à droite et à gauche) par rapport à un banal vibraphone, ou le "piano préparé » de John Cage face à un piano ordinaire. C'est la présence de la chanterelle au milieu des cordes moyennes du théorbe ou entre les deux cordes du gumbri gnawa et du komuz kirghiz, avec les doigtés qui en résultent, ou encore la présence du bourdon entre les deux autres cordes du saz turc (descendant du komuz), elles mêmes doublées à l'octave. Cette astuce multiplie les possibilités «d'accords» les plus inattendus, lesquels proviennent de la position de la main et pas du tout d'une représentation abstraite des hauteurs.

$\mathrm{Au}$ sens technique, l'habileté, c'est l'adéquation et la spécificité du geste lui-même, éventuellement sur un instrument configuré d'une manière particulière, de sorte que les figures deviennent malaisées ou impossible à reproduire sur un autre instrument. Sous cet angle on peut envisager un classement des instruments en hétérogènes vs homogènes. Dans les premiers, les formes musicales sont pré-programmées, ce qui leur donne une efficacité redoutable, évidemment contrebalancée par leurs limitations. Au contraire, les instruments « homogènes » sont moins spécifiques et s'adaptent à de nombreux styles. A cette catégorie appartiennent les flûtes et la plupart des aérophones courants, les vièles comme le violon ou le kamânche, les instruments à claviers (représentant probablement le cas extrême) et la harpe ou le cymbalum dont toutes les notes ont rigoureusement le même statut. Malgré tout, c'est toujours le contact, la prise en main, le geste, le tour de main qui priment, et bien que ces instruments demandent plutôt de la virtuosité, ils autorisent également une certaine "habileté » (laquelle est souvent inspirée d'autres instruments plus hétérogènes) ${ }^{32}$. 
70 Une différence essentielle est aussi que, si la virtuosité mobilise toutes les ressources nerveuses et musculaires, l'habileté au contraire suit les lois du moindre effort et de l'astuce. Elle ne laisse pas d'hiatus entre l'idée, le geste et la forme, de sorte que le musicien, libéré des contraintes et du stress physique, peut s'impliquer sur les autres plans.

71 Les dombra ou dotâr (accordés en quarte ou quinte et montés de frettes chromatiques), appartiendraient plutôt par leur configuration au genre homogène. Ceci n'est vrai que si l'on en joue à la façon du tanbur centre-asiatique, turc ou arabe, en égrenant les notes une à une, de l'index ou du plectre. Dès que l'on fait résonner la basse, ce qui se passe dans le jeu de la main droite, l'instrument devient hétérogène. En effet, avec une basse constante sol, on aura à vide sol-do, puis sol-ré, puis, presque dissonant, sol-mib, et plus encore sol-fa avant d'arriver à l'octave sol-sol, et ainsi de suite. Chaque note n'a pas le même poids ou la même couleur, ce qui se reflète dans les qualificatifs qu'on leur donne parfois : zar parde, shâh parde, etc. (frette d'or, frette royale, dans la terminologie tadjik). C'est pourquoi aussi les musiques nomades refusent cette hiérarchie des degrés (image sédentaire de la hiérarchie ? $)^{33}$ pour changer la basse en fonction des besoins de chaque note : il en résulte une biphonie dont la forme la plus simple procède par quartes parallèles, la plus complexe tissant un véritable contrepoint à deux voix où la mélodie passe d'une corde à l'autre, de même que la ligne secondaire. Ici l'habileté consiste à exploiter toutes les possibilités sans davantage d'effort, puisqu'on ne fait que prendre ce qu'on a sous la main. $\mathrm{Au}$ contraire la virtuosité tente l'impossible, elle «s'étale», parcourt l'espace strié des gammes, comme cela se fait dans la musique occidentale ou indienne.

L'autre facteur hétérogénique, c'est, comme on l'a vu, le jeu de la main droite dont les techniques varient considérablement d'une tradition à l'autre. Au regard de cette richesse, la logique binaire du plectre apparait un peu comme la réduction numérique d'une représentation analogique. On y perd les nuances de timbre propres à chacun des cinq doigts, sans parler de leur réunion possible en une frappe simultanée, par exemple une frappe (index) + majeur + annulaire (+ auriculaire). On y perd aussi la rapidité foudroyante de certaines attaques : frappes légèrement arpégées, doubles frappes, shor, roulements, etc. On y gagne en clarté et en précision, mais au prix d'un temps - et d'un espace - tramé, strié, qui peut apparaitre relativement grossier au regard de l'espace lisse et hétérogène qui est celui de la main entière. 


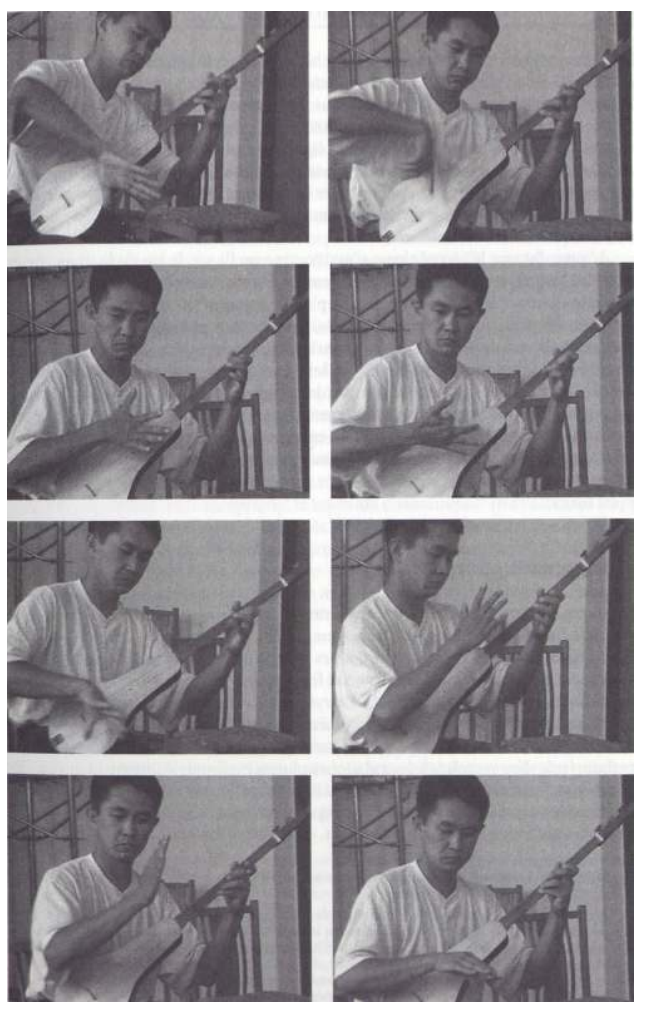

Photo de Jean During

\section{Deux approches du rythme}

Tous ces éléments contribuent à définir ce que l'on qualifie ici d'esthétique nomade. Selon la même logique technique, le jeu des luths se réduit au binaire dans les cultures urbaines et sédentaires, alors qu'elle éclate en ternaire dans les campagnes du Khorassan et du Kurdistan et finit par déborder toute formulation dans les cultures de tradition nomade ${ }^{34}$. Le rythme suit cette même logique. Le geste sédentaire produit une rythmique régulière, prévisible, cyclique, ce qui autorise la synchronie et donc la constitution d'ensembles. Le geste nomade engendre un rythme irrégulier (accelerando, rubato, etc.) non cyclique ; il intègre bien des éléments réguliers, comme ailleurs, mais la libération du geste favorise la succession de formules asymétriques (5, 7, 9 temps) et, plus encore, le mélange imprévisible de toutes sortes de mètres ou, mieux dit, de diverses formules gestuelles. En effet c'est bien la combinaison de "tours de main » variés, donnant des figures de 2, 3, 4, 5 , 6 temps qui est à la source de la rythmique nomade. Ce sens rythmique est certainement issu du geste, du mouvement des doigts et de la main qui, par sa forme, ses possibilités et son usage est loin d'être symétrique comme l'est un plectre. En tous les cas le rythme n'émane pas de la danse ou de la métrique poétique (quantitative, 'aruzi) comme chez les sédentaires türks et indo-iraniens, les poètes nomades ne pratiquant guère ce type de mètre ${ }^{35}$. Les arguments ne manquent pas. Si ce sens rythmique reposait sur une idée, une représentation abstraite ou schématique, on devrait le retrouver dans d'autres expressions musicales n'utilisant pas les ressources de la main droite, comme les 
flûtes ou les vièles. Or précisément, sur ces instruments, les mélodies sont du genre aria libre au flux lent et ample, tout à fait à l'opposé des compositions pour luth.

De l'autre côté, chez les sédentaires d'Asie centrale, danser se traduit par le terme «jouer" (oyna-, raqs onynamôq, raqs tushmôq), mais le concept de danse lui-même se confond avec celui de osul (lit. «les principes») terme technique qui dans la théorie moyen-orientale désigne uniquement le cycle rythmique. Il serait vain de chercher quelque relation de ce genre dans les cultures nomades.

En effet, la danse n'est pas le fort des nomades türks, pas plus que le jeu des instruments de percussion. Brièvement dit, on ne danse point chez les nomades, et inversement, si l'on danse, c'est qu'on est sédentaire, ou sédentarisé de longue date. Il existe cependant deux exceptions: la tradition chamanique ${ }^{36}$ (où l'officiant frappe le tambour, chante et danse), et le jeu du luth lui-même. C'est au point que dans le komuz kirghiz, la gestuelle revêt un caractère esthétique autonome. C'est alors la main qui danse, parfois les deux mains ensemble. Cette danse de la main met en œuvre tout un répertoire de gestes que nous tenterons de classer maintenant.

\section{L'impensable légèreté de la main}

76 On commencera par ranger d'un côté tous les gestes qui affectent un tant soit peu le timbre de l'instrument ou l'interprétation d'une pièce. Autrement dit, tous ceux que l'on pourrait idéalement distinguer des autres à l'audition seule. La palette est très étendue : depuis l'usage de tel ou tel doigt (l'index en descendant, le pouce en montant, quatre doigts ensemble, un léger arpège, une pichenette sur la table, etc), jusqu'à l'amplitude d'un mouvement, la position de la main ou d'un doigt (sur le côté, avec la pulpe ou l'ongle, pincé ou buté, slaping, tapping, etc.). Mais l'oreille a ses limites et même le connaisseur est loin de discerner toutes les nuances de timbre produites par certains mouvements peu courants. Au milieu du registre, se trouvent donc des gestes qui, du point de vue strictement musical, ne sont pas pertinents, mais qui se traduisent tout de même en sons.

En poursuivant cette gradation on arrive à un registre de formes purement gestuelles, qui n'affectent pas le son. Il ne s'agit pas du tout d'une amplification des mouvements standards, comme cela se trouve dans le jeu des pianistes romantiques, et que l'on peut qualifier de gestes expressifs (par exemple la main qui se lève plus haut que nécessaire pour frapper les accords conclusifs d'un morceau, ou les balancements de la tête ou du corps pour s'imprégner de la dynamique d'une musique). En Asie centrale, les gestes du joueur de luth sont rarements expressifs, ils n'expriment que la maitrise technique, le plaisir de les faire joliment, et peut-être de se libérer une seconde des contraintes de l'exécution : geste gratuit qui se donne seulement à voir, ou qui fait voir quelque chose de la musique.

Ainsi, certains gestes semblent matérialiser la vibration : les chanteurs ouzbeks et tadjiks de katta ashula tiennent une petite soucoupe près de leur bouche, comme pour diriger le son dans certaines notes. Geste symbolique, la soucoupe figurant un tambourin, ou efficace à l'instar de la main qui se place naturellement sur le côté de la bouche pour lancer un appel au loin? On ne saurait en juger à l'oreille. Pas davantage du geste du joueur de cymbalum (chang) qui, pour abréger une résonance indésirable, attrape le son qui s'élève de la table d'harmonie en emprisonnant l'air dans ses mains. Effet 
imperceptible mais geste convaincant pour l'interprète et suggestif pour l'auditeurspectateur.

Un degré de plus et l'on entre dans le champ de l'allégorie : la main frappe les cordes du luth et aussitôt semble suivre le mouvement invisible d'une ample onde sonore qui se propage dans l'air. Deux secondes après, elle revient sur l'instrument et reprend son activité musicale. Une autre attaque est suivie d'un revers rapide de la main, comme pour chasser une mouche.

Dans l'air kazakh Buqtum buqtum ${ }^{37}$, un curieux geste se répète de temps en temps, au gré d'une note longue. L'histoire dit que, tout en jouant, le compositeur (ou improvisateur) attirait l'attention de son hôte sur le fait que son verre était vide et qu'il fallait le remplir. Ce dernier n'a pas dû comprendre, sinon la composition traduirait en fin de compte le geste du musicien vidant son verre d'un trait. En tout cas, le geste fait partie de la genèse de la composition. Voici donc une piste d'investigation, ou plutôt une catégorie de plus : il est vrai que les kui et kuu kazakhs et kirghiz ont tous une histoire, un argument (parfois plusieurs, selon les écoles), et que certains gestes s'expliquent ainsi. Eshmambet Moldokunov, paysan de Atbashi, a composé un air « didactique » (nasikat) dans lequel un vieux sage s'adresse aux jeunes. Au cours d'un motif de trois notes égrenées sur le komuz par la main gauche seule, en legato, il caresse tranquillement sa barbe longue et fine de l'autre main, puis les deux côtés d'une moustache kirghiz que le geste suggère bien plus longue et fournie qu'elle ne l'est. S'inquiéter de ce que devient cet air joué par les jeunes gens et les femmes n'est pas une plaisanterie ; il est bien probable qu'en dehors de leur contexte $^{38}$, bien des gestes signifiants soient devenus gratuits, ou simplement esthétiques. Dans l'ensemble pourtant, la gestuelle kazakh et kirghiz semble se suffire à elle-même. Il s'agit de toucher l'auditeur-spectateur, de l'étonner, de l'impressionner, de l'envoûter comme si l'on était un magicien. Dans un kui de dombra, la main frappe ostensiblement la corde sur une note d'un motif ; puis, à la reprise, elle effleure le rebord de la table tandis que le son est émis en pizzicato par la main gauche. On continue en touchant d'autres parties de l'instrument, faisant croire que de partout il peut émettre des sons. Et de fait, en croisant les bras, on arrive à pincer la corde de l'autre côté, derrière la case pressée par le doigt de la main. Comment est-ce possible? Mais bientôt le kui dévoile le stratagème : la main droite se promène partout tandis que la musique continue de la main gauche ; elle grimpe sur le manche, sur la tête de l'instrument et finit dans le vide, comme un oiseau battant de l'aile, sous les applaudissements et les rires.

Dans certaines pièces, les mains entraînent avec elles les bras, puis le corps entier du musicien. Se transformant en cavalier, il chevauche son instrument, et soudain le brandit comme un fusil, le pointe en l'air ou en bas, puis lui fait faire des pirouettes, sans que la mélodie s'interrompe un instant. Les gestes et les façons les plus invraisemblables de manier le komuz sont déclinés sous les yeux du spectateur sidéré. La plupart sont reproduits symétriquement: à l'endroit, à l'envers, à droite à gauche, au-dessus, en dessous, devant, derrière (on en joue dans le dos), main droite, main gauche, puis vice versa, de bas en haut, de haut en bas...

Toutes ces manifestations gestuelles tant musicales que para- et extramusicales révèlent une spécificité de la culture nomade : un goût prononcé pour l'aspect épique, narratif, héroïque des arts de la performance. Il s'agit de se surpasser afin de dominer le concurrent éventuel ; et pour y parvenir, on opère sur tous les plans à la fois en cumulant les effets. Les nomades sont de ce monde, ils ne cherchent pas à concevoir des transcendantaux; ils croient à des forces cachées parce qu'ils en perçoivent les effets. 
Leur musique n'est pas une invitation à s'arracher au monde, à s'oublier un instant dans l'ivresse de la danse; elle est conçue pour être goûtée les yeux écarquillés, comme le préconisait Stravinsky pour toute musique.

Mais il y a aussi des airs qu'on écoute en fermant les yeux. C'est le cas de ce qu'on appelle là-bas les kuu philosophiques. A la fin d'une soirée animée et joyeuse, Ruslan Jumabaev, le plus fort de sa génération annonce: «c'est un kuu très difficile, je ne l'ai pas encore assimilé, mais je vais quand même le jouer ». Et pour une fois, le komuz parle un autre langage : pas de virtuosité, pas de doigtés particuliers, pas de thème narratif, un tempo lent, une pièce relativement longue, grave et retenue comme un choral de Bach. Ce qu'on appelle en Occident de la musique pure.

\section{BIBLIOGRAPHIE}

DELEUZE Gilles et Félix GUATTARI

1980, Mille plateaux. Paris : Éditions de Minuit.

DURING Jean

1989, Musique et mystique dans les traditions de l'Iran. Paris/Téhéran : Institut français de recherche en Iran.

1993 , Asie centrale. Les maîtres du dotâr. Ouzbékistan - Tadjikistan - Iran (Khorâsân) - Turkménistan. Enregistrements et texte : Jean During. 1 CD AIMP XXVI / VDECD-735.

1997a, « Rythmes ovoïdes et quadrature du cercle ». Cahiers de musiques traditionnelles 10 :

« Rythmes »:17-36.

1997b, Ouzbékistan. L'art du dotâr. Enregistrements et texte : Jean During. 1 CD Ocora C 560111.

1997bc, Turkestan. Komuz kirghize et dombra kazakh. Enregistrements et texte : Jean During (avec une contribution de Sabine Trebinjac). 1 CD Ocora C 560121.

1998, Musiques d'Asie Centrale. L'esprit d'une tradition. Arles : Actes Sud / Paris : Cité de la musique.

2001, L'âme des sons. L'art unique d'Ostad Elahi (1895-1974). Paris : Editions du Relié.

SIALA Mourad

1995, La hadra de Sfax : rite soufi ou musique de fête. Thèse de doctorat. Nanterre : Université de Paris X / Nanterre.

\section{NOTES}

1. Luth à long manche et à deux cordes pincées et frappées par les doigts de la main droite. Les formes et les techniques en sont variées, mais le principe est toujours le même.

2. Au XIX ${ }^{\mathrm{e}}$ siècle, probablement sous l'influence azeri, ils tenaient le târ sur l'avant-bras, le menton touchant la caisse. Cette façon de tenir l'instrument est aussi celles des ashiq azeri jouant le sâz, et très loin à l'est, des Türks Ouïgours jouant le rabâb. Les Persans et les Afghans jouaient ce genre d'instrument posé sur les genoux, comme on le voit dans les miniatures. 
3. Transcrit ainsi, il s'agit des Turcs orientaux, non de ceux de Turquie.

4. Hormozi (1897-1976), Sabâ (1902-1957) et Bahâri (1905-1994) sont des maîtres iraniens.

5. Malgré cela ils pourraient bien avoir adopté des traits des cultures nomades türks tout comme ils ont fini par turquiser leur langue. Le clivage nomade / sédentaire ou türk / indo-iranien s'agrémente de bien des accommodements.

6. C'est le cas des bardes épiques du kirghiz cantillant a capella l'épopée Manas. Cf. note 12.

7. Cf. l'analyse détaillée de ces rythmes dans During 1997a.

8. Cette hypersensibilité aux nuances de timbre, à la qualité de la frappe a été relevée dans le contexte de la transe par Mourad Siala dans sa thèse (Siala 1995). La moindre faiblesse de la part du percussionniste peut se traduire par l'échec de l'entrée en transe Selon l'auteur de cette étude, la différence entre musique de transe et d'agrément ne réside que dans la rigueur et l'intensité de l'interprétation, en particulier sur les plans acoustique et rythmique.

9. Le terme étant persan (« deux cordes ») on s'en tient ici à la prononciation persane, laissant de côté les formes dutâr, dutor, duttar, etc.

10. Sur ce point, $c f$. During $1989: 283$.

11. Luth à long manche, de taille moyenne, monté de deux cordes dont l'aiguë est doublée. Son setâr avait une toute petite caisse et comportait quatre cordes.

12. Charokh Elâhi, comm. perso. in During, 2001.

13. Inspirées entre autres par des conversations avec le regretté Nâser Farhangfar, génial joueur de zarb et brillant calligraphe.

14. En particulier dans l'art de l'épopée kirghiz, Manas, qui se fait en cantillant rythmiquement, sans instrument, mais avec une riche gamme de gestes expressifs ou mimétiques.

15. Ce concept provient selon nous dans le jeu de la harpe (chang), disparue depuis le XVII ${ }^{\mathrm{e}}$ siècle en Asie intérieure. Sur cet instrument les notes fortes étaient jouées par la main droite et les faibles par la gauche (chang signifie en persan griffe ou main ouverte comme une griffe). Ce principe vaut aussi pour le santur (cymbalum), qui, ce n'est pas un hasard, est appelé chang en Asie centrale. Le jeu des percussions pourrait aussi être à l'origine de cette distinction, puisque dans le tambour sur cadre, les coups forts sont faits par la main droite. Ainsi le jeu du tanbur kurde reproduit exactement celui du tambourin: les coups frappés par la main droite correspondent à une attaque des cordes de haut en bas (coup droit) et inversement pour la main gauche (coup gauche). Sur les deux instruments les remplissages ou monnayages (shor et riz) sont à la même place du cycle rythmique.

16. Les autres écoles de tanbur kurde, d'un niveau nettement moins sophistiqué, n'utilisent pas le pouce, et entrent donc dans la catégorie des instruments à monnayage binaire.

17. Selon le maitre ouzbek Turgun Alimatov, le dotâr est antérieur au tanbur centre-asiatique (luth à long manche aux cordes pincées par l'index droit muni d'un onglet).

18. L'importance accordée à l'articulation correcte, c'est-à-dire à la juste place des coups ${ }^{\wedge}$ et $v$ dans le târ, le santur et le setâr persan, a probablement son origine dans le jeu d'instruments comme la harpe et le dotâr. De plus, le répertoire canonique persan (radif), dans ses versions pour instrument à plectre (mezrâb), abonde en figures disons "chiro-rythmiques» qui se placent indifféremment dans diverses mélodies types (gushe). Ces formules ne se trouvent pas dans le jeu du luth turc ou arabe, elles n'ont aucun rapport avec le chant ou le mètre poétique, et elles sont loin d'émaner naturellement de la simple ornementation d'une ligne de chant ou du remplissage d'un cycle rythmique (car elles ne sont pas «mesurées »). Elles ont été élaborées de façon autonome sur l'instrument, dans la même logique que les doigtés de la main droite pour les luths centre-asiatiques, et dans certains cas, en constituent probablement une adaptation.

19. Style shertpe (pincé) du Centre et du Sud, topke (frappé) de l'Ouest. Les deux techniques sont fondues dans le jeu kirghiz.

20. En effet, la percussion n'ajoute que son timbre propre, elle ne subdivise pas la mesure de façon plus fine ou variée que le dotâr. Inversement le frottement volontaire des doigts sur la 
caisse, ou encore des pichenettes, font du dotâr un idiophone occasionnel. Aussi, même dans les airs bien mesurés des Ouzbeks, le dotâr en solo n'est jamais accompagné de percussion. Cela se vérifie aussi chez les Kurdes Ahl-e haqq, qui traditionnellement jouent le tanbur sans percussion. Ce trait se retrouve en Anatolie dans le jeu du saz. Chez les Baloutches également, la percussion est superflue du fait que le rythme est donné par le tanburag, une autre variété de dotâr. Chez les Ouïgours d'Ili, la combinaison traditionnelle tanbur (pincé à l'onglet) et dotâr exclut tout instrument de percussion. L'absence de percussion n'est donc pas nécessairement un trait des cultures nomades, elle est également lié à la présence du dotâr.

21. On distingue ceux qui sont spécialisés dans le solo (kuichi) de ceux qui chantent en s'accompagnant du luth (erchi, akin, etc.)

22. Au Kazakhstan, le barde épique avait traditionnellement un rôle de conseiller du khan. De nos jours encore, certains ont des responsabilités politiques.

23. Les duos (barde jouant du dotâr + vièle) se trouvent chez les Turkmènes et Qâraqalpâk, mais le barde a de loin le premier rôle, la vièle se contentant de souligner le chant. Lorsque la balance s'établit entre les deux, comme chez les Ouïgours de Ili (chant, dotâr, tanbur), c'est qu'on n'est plus en milieu nomade.

24. Soit par des monnayages, comme on l'a vu plus haut, soit par des raclements des doigts sur la table. Dans le jeu du tanburag baloutche, certains parviennent à percuter la table tout en jouant, de façon à reproduire la battue syncopée d'un tambour biface (dohol). On peut évidemment aussi frapper sur la table entre les notes ou à leur place.

25. On note que dans le jeu de guimbarde ouzbek (Bôysun, Sorkhandaryâ), la biphonie est nettement moins présente que chez les autres peuples Türks. Elle consiste en effets acoustiques et rythmiques, elle sert éventuellement à faire danser, à imiter la parole. Le modèle nomade s'estompe dans cette région peuplée depuis l'antiquité de sédentaires.

26. Par exemple " regardant, il resté » = il surveille, " étant vendu, prenez » = achetez, « ayant pris (ou prenant), je viens » = j'apporte.

27. Du point de vue technique, on retrouve les mêmes contrastes: le sitâr se joue avec deux doigts de la main gauche et à l'aide d'un onglet à la main droite, le dombra kazakh ou le tanbur kurde, utilise jusqu'aux cinq doigts de chaque main.

28. Le modèle que nous esquissons ne prétend pas dépasser l'aire géographique en question. Nous laissons aux specialistes d'autres cultures le soin de juger s'il est de quelque pertinence ailleurs. Quoiqu'il en soit il présente son intérêt propre.

29. Les termes entre guillemets sont empruntés à Deleuze et Guattari 1980.

30. Cf. During 1997a : 31-33, et 1998, chapitre I, où l'on a appliqué ces concepts non plus à l'espace, comme leurs inventeurs, mais au temps. On arrive ainsi au concept de «temps nomade » tout différent de celui des horloges et des métronomes.

31. Certains objets artisanaux en donnent une illustration : les ligatures qui assemblent la lame d'une hache sur son manche, par exemple, et dont la fonction (faire tenir l'assemblage) devient secondaire par rapport à l'adéquation, l'habileté et l'élégance des entrelacs, si bien que le moyen fait oublier la fin et devient art.

32. C'est par exemple, d'un point de vue technique, les sauts d'archet dans les airs de violon ou de violoncelle de Bach qui donnent l'illusion de deux voix alors qu'il n'y en a qu'une, ainsi que tous les doigtés qui, dans des musiques fondamentalement monodiques, produisent un effet analogue. 33. On pense à la gamme chinoise qui symbolise la hiérarchie d'Etat, avec l'Empereur, le premier ministre, etc.

34. Ainsi, au contact de la ville et de la musique de l'Inde, le dotâr afghan s'est coupé de ses origines rurales, et l'usage des doigts s'y est perdu. On le pince avec un onglet selon le système binaire du rubâb. Les grands dotâr ouïgour ainsi que tadjik sont également plus "sédentarisés " (on veut dire plus " posé », plus simple) que le dotâr ouzbek, d'ailleurs leur taille énorme permet difficilement de les transporter. Chez les Ouzbeks, cet instrument apparaît comme lié à la steppe, 
à la culture nomade, même s'il est totalement intégré à la musique d'art. Pour aller plus loin dans la complexité de jeu, il suffit de se tourner vers des peuples sédentarisés bien plus tard que les Ouzbeks, tels que les Kirghiz et les Kazakhs.

35. Dans toute l'Asie centrale, on trouve couramment certains mètres classiques, du genre - $\mathrm{u}$ - - / (sept temps répété quatre fois), mais il est fort possible qu'il ne s'agisse pas d'emprunts à la prosodie classique, mais de mètres très anciens, qui sont plus probablement issus de la musique que de la prosodie.

36. Dans le cas des chamanes, il s'agit cependant bien plutôt de pulsation que de cycle rythmique. Mais ici comme dans toute classification, il y a des zones intermédiaires, correspondant à des niveaux de sédentarisation. Ainsi, dans un enregistrement très rare d'une séance chamanique ouïgour (enregistré par A. Hashimov dans les années 1970), le tambourin accompagne le chant sur un cycle à six temps lents, comme dans la musique savante. De même, les Turkmènes ont une danse qui imite le zikr et samâ' tel que le font les derviches ouiggours. On trouve aussi des éléments de danse dans les tournois de lutte mongole, donc encore dans un contexte rituel. C'est peu au regard de la place de la danse en Transoxiane, en Afghanistan ou chez les Ouïgours.

37. Composition de Qürmanghazy Saghyrbaiüly (1818-1879). Ces deux mots sont énoncés à travers les sonorités de l'instrument lui-même.

38. On dit qu'à l'origine, les kuu étaient des intermèdes au cours de récits ou d'épopées.

\section{RÉSUMÉS}

Les diverses modalités du geste sont passées ici en revue dans le cadre des cultures de l'Asie intérieure: la posture ou position (le point immobile comme condition du mouvement), le toucher et ses secrets, le rapport au corps et la valeur sémantique du mouvement physique, la réduction des mouvements des cinq doigts à un système binaire de coups de plectre, la grammaire rythmique des luths à deux cordes, etc. De ce survol se dessinent quelques lignes de partage : au niveau conceptuel, entre instruments hétérogènes et homogènes, entre temps lisse et temps strié, entre esthétique de l'asymétrie et de la symétrie, ainsi qu'une esquisse de caté gorisation: geste qui affecte le timbres, qui produit du flux, du rythme ou des formules rythmiques (engendrant la danse), geste expressif qui souligne la ligne mélodique, enfin geste autonome, héroïque, acrobatique du barde épique, suggestif ou simplement chorégraphique du joueur de luth. Les musiciens de tous horizons partagent les mêmes dispositions physiques et les mêmes préoccupations techniques, comme le suggèrent de fréquentes références à la culture musicale de l'Occident. Toutefois, leurs choix esthétiques se traduisent par de profonds contrastes entre, par exemple, l'image du corps durant la performance (statique ou dynamique), le contact avec l'instrument (faisant corps avec soi, tenu à distance, en rapport de cavalier et de monture), les formes rythmiques (issues directement du geste) et le sens du temps qui en découle, la technique de jeu, l'agencement de l'instrument, etc. Au terme d'une étude comparative couvrant les pratiques musicales de ce qu'on appelle l'Asie intérieure, on dégage clairement deux modèles bien distincts susceptibles d'être affinés par les données de l'anthropologie culturelle : nomade et turcique d'un côté, sédentaire et iranien de l'autre. 


\section{AUTEUR}

JEAN DURING

Jean DURING, né en 1947, docteur es Lettres, directeur de recherche au C.N.R.S et directeur de thèse à Paris $\mathrm{X}$, est ethnomusicologue, orientaliste et musicien. Il a publié une dizaine d'ouvrages sur les cultures musicales de l'Asie Intérieure, une centaine d'articles dans des revues scientifiques et des encyclopédies, et deux douzaines de CD. De 1971 à 1981, il a séjourné en Iran, étudiant la pratique instrumentale et la philosophie traditionnelle. Il réside depuis trois ans en Asie Centrale, en détachement et en missions de longue durée, réalisant des programmes de recherche et de sauvegarde pour le compte de l'Aga Khan Music Initiative in Central Asia. Son champ de recherche couvre les cultures turciques et iraniennes depuis l'Azerbaïdjan jusqu'au Xinjiang. 